\title{
Intestinal Permeability Regulation by Tight Junction: Implication on Inflammatory Bowel Diseases
}

\author{
Sung Hee Lee \\ Institute of Pharmaceutical Research and Development, Wonkwang University College of Pharmacy; BK21plus program \& Department of \\ Smart Life-Care Convergence, Wonkwang University Graduate School, Iksan, Korea
}

Epithelial tight junctions (TJs) are the key structures regulating paracellular trafficking of macromolecules. The TJ is multiprotein complex that forms a selective permeable seal between adjacent epithelial cells and demarcates the boundary between apical and basolateral membrane domains. Disruption of the intestinal TJ barrier, followed by permeation of luminal noxious molecules, induces a perturbation of the mucosal immune system and inflammation, which can act as a trigger for the development of intestinal and systemic diseases. Inflammatory bowel disease (IBD) patients demonstrate increased intestinal paracellular permeability. Although it remains unclear whether barrier dysfunction precedes disease or results from active inflammation, increased intestinal TJ disruption is observed in IBD patients suggest that dysregulation of TJ barrier integrity may predispose or enhance IBD progression. Therefore, therapeutic target to restore the TJ barrier integrity may provide effective therapeutic and preventive approaches against IBD. This review discusses the molecular structure and regulation of intestinal TJs and the involvement of intestinal TJs in IBD pathogenesis. (Intest Res 2015;13:1 1-18)

Key Words: Intestinal permeability; Tight junctions; Inflammatory bowel diseases; Intestinal barrier function; Paracellular permeability

\section{INTRODUCTION}

Intestinal permeability is the property that allows solute and fluid exchange between the lumen and tissues. Conversely, intestinal barrier function refers to the ability of the mucosa and extracellular barrier components, such as mucus, to prevent this exchange.

A critical function of the intestinal epithelium is to form a barrier that prevents permeation of pro-inflammatory molecules, such as pathogens, toxins, and antigens, from the luminal environment into the mucosal tissues and circulatory system. ${ }^{1}$ Barrier defects have been associated with a variety

Received August 18, 2014. Revised August 20, 2014.

Accepted August 21, 2014.

Correspondence to Sung Hee Lee, Wonkwang University College of

Pharmacy, 460 Iksan-daero, Iksan, Jeollabuk-do 570-749, Korea. Tel: +82-

63-850-6820, Fax: +82-63-854-6038,E-mail: gsseo@wku.ac.kr

Financial support: This study was supported by a grant from Wonkwang

University 2013. Conflict of interest: None. of human diseases, including those primarily affecting the gut, such as IBD, celiac disease, and IBS, as well as systemic diseases or diseases involving other organ systems, such as type I diabetes, acquired immunodeficiency syndrome (AIDS), multiple sclerosis, and rheumatoid arthritis. ${ }^{2-7}$

Epithelial tight junctions (TJs) maintain the intestinal barrier while regulating permeability of ions, nutrients, and water. The TJ is a multi-protein complex that forms a selectively permeable seal between adjacent epithelial cells and demarcates the boundary between apical and basolateral membrane domains. ${ }^{8}$ The modification of TJ barrier function and paracellular permeability is dynamically regulated by various extracellular stimuli and is closely associated with our healthy and susceptibility disease. ${ }^{1,9}$ TJ barrier disruption and increased paracellular permeability, followed by permeation of luminal pro-inflammatory molecules, can induce activation of the mucosal immune system, resulting in sustained inflammation and tissue damage.

Evidence from basic science and clinical studies indicate

๑ Copyright 2015. Korean Association for the Study of Intestinal Diseases. All rights reserved.

This is an Open Access article distributed under the terms of the Creative Commons Attribution Non-Commercial License (http://creativecommons.org/licenses/by-nc/3.0)

which permits unrestricted non-commercial use, distribution, and reproduction in any medium, provided the original work is properly cited. 
that the intestinal TJ barrier has a critical role in the pathogenesis of intestinal and systemic diseases. ${ }^{1,10}$ Thus, this review summarizes the molecular structure and regulation of intestinal TJs and the involvement of intestinal TJs in IBD pathogenesis.

\section{STRUCTURES AND FUNCTIONS OF THE TJ PRO- TEINS}

TJs are multiple protein complexes located at the apical ends of the lateral membranes of intestinal epithelial cells (Fig. 1). ${ }^{11,12}$ Four integral transmembrane proteins, occludin, ${ }^{13}$ claudins, ${ }^{14}$ junctional adhesion molecule (JAM) ${ }^{15}$ and tricelluin, ${ }^{16}$ have been identified. The intracellular domains of these transmemebrane proteins interact with cytosolic scaffold proteins, such as zonula occludens (ZO) proteins, which in turn anchor the transmembrane proteins to the actin cytoskeleton. The interaction of $\mathrm{TJ}$ proteins with the actin cytoskeleton is vital to the maintenance of TJ structure and permits the cytoskeletal regulation of TJ barrier integrity. ${ }^{17}$ The circumferential contraction and tension in the actin is regulated by myosin light chain (MLC) activation. ${ }^{18}$ Induction of MLC phosphorylation by kinases such as MLC kinase (MLCK) and Rho-associated

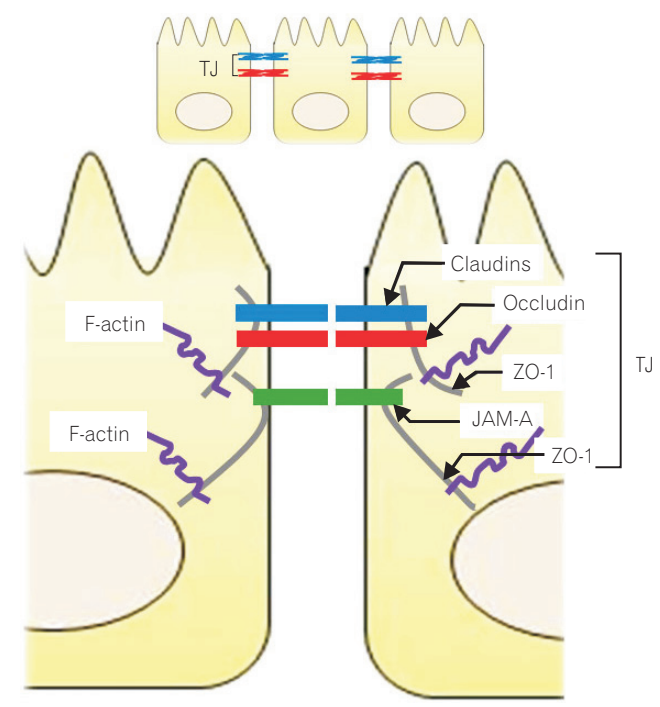

Fig. 1. Molecular structure of the intracellular junction of intestinal epithelial cells. The tight junctions (TJs), multiple protein complexes, locate at the apical ends of the lateral membranes of intestinal epithelial cells. The TJ complex consists of transmembrane and intracellular scaffold proteins. The transmembrane proteins (claudins, occludin, and junctional adhesion molecules [JAMs]) create a permselective barrier in the paracellular pathways. The intracellular domains of the transmembrane proteins interact with the intracellular scaffold proteins such as zonula occludens (ZO) proteins, which in turn anchor the transmembrane proteins to the actin cytoskeleton. kinase causes the contraction of the actin, resulting in the opening of the paracellular pathways. ${ }^{18,19}$

\section{Occludin}

Occludin was the first integral membrane TJ protein. ${ }^{13}$ The long C-terminal domain of occludin interacts with several intracellular TJ proteins, such as ZO proteins, which are required to link occludin to the actin cytoskeleton (Fig. 2). ${ }^{20}$ The function of occludin is not yet fully understood, but numerous studies using animals and cell cultures indicate that it has crucial roles in the TJ structure and permeability in the intestinal epithelia. $^{21}$

Recent studies showed that knockdown of occludin induces an increase in paracellular permeability to macromolecules, which indicates that occludin plays a role in the maintenance and assembly of TJs. ${ }^{21}$ In the intact epithelium, occludin is highly phosphorylated on the serine and threonine residues, ${ }^{22}$ and the phosphorylation has a role in the maintenance and assembly of the TJ structure. ${ }^{23}$ Some kinases, such as protein kinase C (PKC) and casein kinase, have been identified as responsible for its phosphorylation. ${ }^{24,25}$ In addition, although the level of tyrosine-phosphorylated occludin is very low in the intact epithelium, several studies have shown that tyrosine phosphorylation is caused during disassembly by various stimuli. ${ }^{26}$

Underlying this mechanism, the tyrosine phosphorylation of occludin attenuates the interaction with ZO-1, leading to dissociation from the junctional complex. Like hydrogen peroxide, acetaldehyde, a biological metabolite of ethanol,

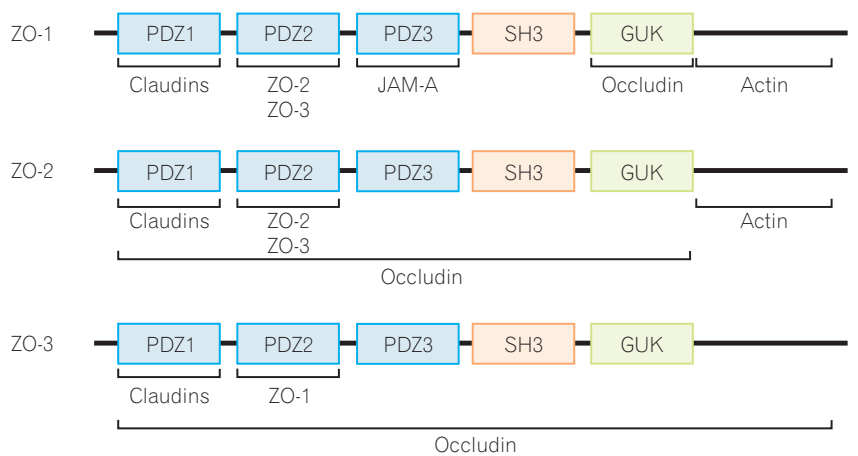

Fig. 2. Interaction of proteins with the integral scaffold tight junction (TJ) proteins, zonula occludens (ZO)-1, -2 , and -3 . Z0 proteins carry 3 post-synaptic density 95/Drosophila disc large/zona-occludens (PDZ) domains, a Src homology-3 (SH3) domain, and a region of homology to guanylate kinase (GUK) from the side of the N-terminus. Several TJ proteins and cytoskeletal actin interact with the ZO proteins. JAM-A, junctional adhesion molecule-A. 
also induces TJ disruption in intestinal epithelial cells in an occludin tyrosine phosphorylation-dependent manner. ${ }^{27}$

\section{Claudins}

Claudins do not have any sequence similarity to occludin. The extracellular loops of claudin molecules make hemophilic and heterophilic interactions with adjacent cells and the interactions create either barriers against or pores for the passage of selective molecules in the paracellular pathways. ${ }^{12,28}$ Numerous studies have demonstrated that claudins are the key component and backbone of TJs. When claudins are expressed in fibroblast, they are incorporated into TJ strands and form paired strands at the cell-cell contacts. ${ }^{14}$

Recent studies using claudin knockout mice have shown that claudins play a critical role in barrier formation and paracellular permeable selectivity in various tissues. ${ }^{29}$ As a prominent example, claudin-1 knockout mice die within 24 hours of birth because of a dramatic loss of fluid and electrolytes through the impaired epidermal barrier. Claudins are a multigene family with at least 24 members in humans and mice, and each isoform shows a unique expression pattern in tissues and cell lines. As is the case with occludin, some claudin isoforms are phosphorylated in the cells, ${ }^{30}$ and this phosphorylation is associated with localization and paracellular permeability.

\section{Junctional Adhesion Molecule}

The JAM family belongs to the Ig superfamily and is characterized by 2 extracellular Ig domains, one transmembrane domain, and one intracellular C-terminal domain. ${ }^{31}$ The extracellular N-terminal domains of the JAM family members bind to various ligands through hemophilic and heterophilic interactions. ${ }^{32}$ The hemophilic interactions involving the JAM members have a role in the formation of TJs and the cell-cell border. In contrast, the heterophilic interactions function in cell-cell adhesion, association between leukocytes and epithelial/endothelial cells, platelet activation, and virus recognition.

JAM members are expressed in various cell types including epithelial, endothelial, and immune cells, and exhibit different expression patterns in both a tissue- and cell typespecific manner. In intestinal epithelial cells, JAM-A and JAM-4 are expressed and involved in TJ regulation. In vitro and in vivo studies demonstrate that JAM-A participates in the regulation and maintenance of the TJ barrier. Treatment of intestinal epithelial cells with monoclonal JAM-A antibod- ies inhibited the resealing of the TJs, as indicated by delays in transepithelial electrical resistance (TER) recovery and occludin assembly. ${ }^{33}$ Recent studies have shown that JAM-A knockout mice exhibit higher permeability to dextran and myeloperoxidase activity in the colon compared to wild type mice. In addition, the colonic injury and inflammation induced by dextran sodium sulfate are more severe in the JAM-A knockout mice than in wild type mice. ${ }^{34}$

\section{Zonula Occludens}

The $\mathrm{ZO}$ proteins were the first TJ-specific proteins identified and three ZO proteins, ZO-1, -2 , and -3 , have been identified to date. ${ }^{35}$ According to sequence analysis, these $\mathrm{ZO}$ proteins are categorized as members of the membraneassociated guanylate kinase homolog family. ${ }^{17}$ They are multi-domain proteins carrying three post-synaptic density 95/Drosophila disc large/zona-occludens (PDZ) domains, a Src homology-3 domain and a region of homology to guanylate kinase homolog from the side of the N-terminus (Fig. 2). ${ }^{36}$ These multi-domain structures provide an intracellular scaffold in the TJs and are required for regulation and maintenance of TJ structure.

Interestingly, many $\mathrm{TJ}$ proteins bind to the N-terminal half region of $\mathrm{ZO}$ proteins, while the $\mathrm{C}$-terminal region interacts with the actin cytoskeleton and cytoskeleton-associated proteins. ${ }^{37}$ Among the $\mathrm{ZO}$ proteins, the biochemical function and property of ZO-1 have been well examined. ZO-1 localizes to the nascent cell-cell contacts in both cell cultures and animal models. Therefore, it has been proposed that ZO proteins may mediate the early assembly of TJ proteins into cell-cell contacts. To data, intensive efforts have been made to clarify the functional role of $\mathrm{ZO}$ proteins, but it has been difficult to obtain clear evidence showing the importance of $\mathrm{ZO}$ proteins in $\mathrm{TJ}$ regulation. Recent studies have been shown that ZO-1 deficient cells are still able to form normal TJ structures and show normal permeability; however, an obvious delay in the assembly of other TJ proteins including occludin and claudins into the TJ is observed, indicating that the $\mathrm{ZO}$ proteins have an important role in the regulation of TJ assembly. ${ }^{38}$

\section{REGULATION OF THE TJ PROTEINS}

The roles of cytokines in intestinal $\mathrm{TJ}$ regulation under pathophysiological conditions have been well investigated. The cytokine-mediated dysfunction of the TJ barrier, resulting in immune activation and tissue inflammation, is thought 
to be important in the initiation and/or development of several intestinal and systemic diseases. ${ }^{1,10}$ In contrast, some growth factors play roles in protection and maintenance of TJ integrity.

\section{Interferon- $\gamma$}

Interferon- $\gamma$ (IFN- $\gamma)$ is mainly involved in the regulation of inflammatory immune responses, and levels are elevated in the intestinal mucosa in patients with IBD. ${ }^{39}$ Recent studies have also demonstrated that IFN- $\gamma$ increases paracellular permeability in intestinal epithelial cells through the redistribution and expression of TJ proteins and the rearrangement of the actin cytoskeleton. ${ }^{40}$ Thus, IFN- $\gamma$ increases actinmyosin contractility in a Rho-associated kinase-dependent manner and induces TJ protein internalization, resulting in intestinal TJ disruption.

\section{Tumor Necrosis Factor- $\alpha$}

Tumor necrosis factor- $\alpha$ (TNF- $\alpha$ ) is a proinflammatory cytokine that is produced mainly by activated $\mathrm{T}$ cells and macrophages. Clinical studies show that TNF- $\alpha$ is elevated in the intestinal mucosa, serum, and stools of IBD patients. TNF- $\alpha$ is known to induce apoptosis and inflammatory response in intestinal epithelial cells, ${ }^{41}$ and recent studies have also demonstrated that it impairs the intestinal TJ barrier through different mechanism. In intestinal Caco-2 cells, MLCK expression, which has an important role in TJ regulation, was interrelated with the TNF- $\alpha$-induced barrier defect. The TNF- $\alpha$-induced decrease in TER is well correlated with MLCK expression and MLC phosphorylation. ${ }^{42}$ In addition, in intestinal HT-29/B6 cells, TNF- $\alpha$ was seen to decrease TER through claudin-2 expression. ${ }^{43}$ Claudin-2 is expressed throughout the intestine and forms a pore for cations, which is blocked by involvement of phosphatidyl inositol-3 kinase (PI3K)/Akt signaling.

\section{Interleukin-1 $\beta$}

Interleukin- $1 \beta$ (IL- $1 \beta$ ) is markedly elevated in intestinal mucosa under inflammatory conditions, such as CD patients. ${ }^{44}$ In clinical studies, an IL-1 receptor antagonist is currently being developed for therapeutic usage, which indicates IL- $1 \beta$ plays a central role in the intestinal inflammatory process. ${ }^{45}$ Furthermore, recent studies show that IL$1 \beta$ causes increased intestinal TJ permeability. In intestinal Caco- 2 cells, IL- $1 \beta$ decreases TER and increases inulin flux, which is in part mediated by the decreased expression and redistribution of occludin and increased expression of MLCK and MLC phosphorylation. ${ }^{46}$ It was also shown that the knockdown of nuclear factor- $\kappa \mathrm{B}$ p65 inhibits the decrease in occludin and increases in MLCK expression, indicating nuclear factor- $\kappa \mathrm{B}$-dependent transcriptional regulation by IL- $1 \beta$. $^{47}$

\section{Interleukin-6}

The major source of interleukin-6 (IL-6) seen in IBD has been shown to be intestinal epithelial cells and lamina propria mononuclear cells. ${ }^{48}$ Recent studies indicate the involvement of IL-6 in intestinal TJ regulation. ${ }^{49}$ IL-6 increases paracellular permeability selectively to cations, but not to macromolecules, with an increase in pore-forming claudin-2 in intestinal epithelial cells, which requires the mitogen-activated protein kinase (MEK)/extracellular signal-regulated kinase (ERK) and PI3K/Akt signaling activations.

\section{Interleukin-10}

Interleukin-10 (IL-10) is regarded as an anti-inflammatory cytokine. In vitro studies have demonstrated that IL-10 opposes the cellular functions induced by TNF- $\alpha$ and IFN- $\gamma .^{50}$ IL-10 knockout mice, which are widely used as a model of spontaneous colitis, show increased intestinal permeability with elevated TNF- $\alpha$, IL- $1 \beta$, and IL-6 expression prior to exhibiting histologic signs of intestinal inflammation. ${ }^{51}$ These observations suggest that IL-10 has a role in the protection of the intestinal barrier. Clinical and animal studies have demonstrated that a major physiological consequence of total parenteral nutrition is the loss of intestinal barrier integrity. ${ }^{52}$ Mice administrated with total parenteral nutrition show increased intestinal paracellular permeability and decreased levels of TJ proteins, which is reversed by IL-10 treatment. ${ }^{53}$ Collectively, IL-10 restores the intestinal barrier defect.

\section{Interleukin-17}

Interleukin-17 (IL-17) is mainly produced in Th17 cells, potently induces tissue inflammation, and is associated with the pathogenesis a variety of autoimmune conditions, including rheumatoid arthritis, multiple sclerosis, and IBD. ${ }^{54}$ Information regarding intestinal barrier regulation by IL17 is limited, but one study has demonstrated that IL-17 induces claudin expression. ${ }^{55}$ In intestinal T84 cells, IL-17 increases 2 claudin isoforms, claudin- 1 and -2 , which have op- 
posing effects on TJ integrity. The MEK pathway is involved in IL-17-induced claudin-2 expression, but not claudin-1 expression.

\section{Epidermal Growth Factor}

Epidermal growth factor (EGF) is initially synthesized as a precursor transmembrane protein, and the mature and soluble form is released by proteolytic cleavage. The EGF receptor is expressed on the membrane surface of most cell types, and EGF mediates various biological functions, including cellular proliferation, differentiation, and survival through the receptor. Further, recent studies have shown that EGF protects intestinal barrier function against noxious stimuli including oxidative stress, ethanol, and acetaldehyde. ${ }^{56}$ EGF is known to activate several signaling pathways such as PKC and mitogen-activated protein kinase. The activation of the MEK/ERK pathway seems to be required for the EGF-mediated protection of the intestinal barrier against the oxidative stress. Acetaldehyde also disrupts barrier function, which is inhibited by pretreatment of EGF through the activation of EGF receptor-phospholipase- $\gamma$-PKC $\beta 1 / \varepsilon$ and EGF receptorMEK/ERK signaling pathways. ${ }^{57}$

\section{Transforming Growth Factor- $\beta$}

Transforming growth factor- $\beta$ (TGF- $\beta$ ) is a highly pleiotropic cytokine and proposed to act as a cellular switch to regulate immune function, proliferation, and epithelial mesenchymal transition. Several studies have demonstrated that TGF- $\beta$ has protective or promotive effects on intestinal barrier function. ${ }^{58}$ In intestinal T84 cells, TGF- $\beta$ increases the basal TER, which is mediated by claudin- 1 expression through MEK/ERK signaling. In contrast, TGF- $\beta$ protects the intestinal TJ barrier against noxious stimuli including cryptosporidium parvum and enterohemorrhagic Escherichia coli. In T84 cells, enterohemorrhagic $E$. coli disrupts the TJ barrier by disturbing the expression and distribution of TJ proteins, including ZO-1, caludin-2, and occludin, which is attenuated by pre-treatment of TGF- $\beta$.

\section{INVOLVEMENT OF THE TJ IN IBD}

IBD, including UC and CD, are chronic and relapsingremitting inflammatory diseases. Although the exact cause of IBD remains unknown, genetic susceptibility, environmental factors, and immune dysregulation all contribute to disease pathogenesis. In addition, IBD patients demonstrate increased intestinal paracellular permeability, which reflects decreased epithelial barrier function in IBD ${ }^{59}$ Clinical studies show decreased expression and redistribution of barrierforming proteins, including occludin, claudin-3, -5, -8, and JAM-A, and increased expression of pore-forming claudin-2 in CD patients. ${ }^{60,61}$ In UC patients, decreased expression and redistribution of occludin, claudin-1, -4, and JAM-A, and increased caludin-2 expression are observed. ${ }^{61}$ In addition, MLCK expression and MLC phosphorylation are also observed in the intestines of both UC and CD patients, indicating that cytoskeletal dysregulation is involved in the increased intestinal permeability of IBD patients. ${ }^{62}$

Clinical studies show that the levels of inflammatory cytokines, including TNF- $\alpha,{ }^{63}$ IFN- $\gamma,{ }^{39}$ IL- $1 \beta,{ }^{44}$ IL- $6,{ }^{64}$ and IL- $17^{54}$ are elevated in the intestines of IBD patients. As described above, these cytokines are known to disturb intestinal barrier function. In particular, TNF- $\alpha$ may have an important role in the initiation and development of intestinal barrier defects in IBD, because anti-TNF- $\alpha$ treatment suppresses the inflammatory response and improves intestinal permeability. ${ }^{65}$ As a consequence of perturbation of multiple cytokines, the alterations to the intestinal TJ structures in IBD patients could be complicated. In intestinal HT-29/B6 cells, TNF- $\alpha$ treatment resulted in decreased TJ strand number and complexity and increased frequency of strand breaks. ${ }^{66}$ TNF- $\alpha$ also inhibits occludin promoter activity ${ }^{43}$ and causes redistribution of occludin, ZO-1, and claudin- $1 .^{67}$ In vitro and in vivo, transcription and translation of MLCK, the major effector responsible for TNF- $\alpha$-induced TJ modulation, are increased by TNF- $\alpha{ }^{43,67,68}$ Other pro-inflammatory cytokines may also mediate barrier function through modulation of MLCK activity. Since represents a common effector used by multiple cytokines to modulate paracellular permeability, it might be an important target for further therapies to restore barrier function during active disease.

\section{CONCLUSIONS}

Epithelial barrier dysfunction and inflammation are major contributors to the pathogenesis intestinal disease. Intestinal TJ structures are organized by multiple integral proteins and signaling molecules. Clinical and experimental studies demonstrate that defects in the intestinal TJ barrier and increased permeability are observed in various intestinal and systemic diseases. Intestinal TJ barrier is dynamically regulated by cytokines and pathogens. Therefore, understanding the molecular mechanisms underlying TJ regulation by the extracellular factors will lead to the development of effective therapeutic and preventive approaches against diseases associated with intestinal barrier defects such as IBD. 


\section{REFERENCES}

1. Turner JR. Intestinal mucosal barrier function in health and disease. Nat Rev Immunol 2009;9:799-809.

2. van Elburg RM, Uil JJ, Mulder CJ, Heymans HS. Intestinal permeability in patients with coeliac disease and relatives of patients with coeliac disease. Gut 1993;34:354-357.

3. Dunlop SP, Hebden J, Campbell E, et al. Abnormal intestinal permeability in subgroups of diarrhea-predominant irritable bowel syndromes. Am J Gastroenterol 2006;101:1288-1294.

4. Bosi E, Molteni L, Radaelli MG, et al. Increased intestinal permeability precedes clinical onset of type 1 diabetes. Diabetologia 2006;49:2824-2827.

5. Sharpstone D, Neild P, Crane R, et al. Small intestinal transit, absorption, and permeability in patients with AIDS with and without diarrhoea. Gut 1999;45:70-76.

6. Yacyshyn B, Meddings J, Sadowski D, Bowen-Yacyshyn MB. Multiple sclerosis patients have peripheral blood CD45RO+ B cells and increased intestinal permeability. Dig Dis Sci 1996;41: 2493-2498.

7. Martínez-González O, Cantero-Hinojosa J, Paule-Sastre P, Gómez-Magán JC, Salvatierra-Ríos D. Intestinal permeability in patients with ankylosing spondylitis and their healthy relatives. Br J Rheumatol 1994;33:644-647.

8. Turner JR. Molecular basis of epithelial barrier regulation: from basic mechanisms to clinical application. Am J Pathol 2006;169: 1901-1909.

9. Nusrat A, Turner JR, Madara JL. Molecular physiology and pathophysiology of tight junctions. IV. Regulation of tight junctions by extracellular stimuli: nutrients, cytokines, and immune cells. Am J Physiol Gastrointest Liver Physiol 2000;279:G851G857.

10. Farhadi A, Banan A, Fields J, Keshavarzian A. Intestinal barrier: an interface between health and disease. J Gastroenterol Hepatol 2003;18:479-497.

11. Tsukita S, Furuse M, Itoh M. Multifunctional strands in tight junctions. Nat Rev Mol Cell Biol 2001;2:285-293.

12. Van Itallie CM, Anderson JM. Claudins and epithelial paracellular transport. Annu Rev Physiol 2006;68:403-429.

13. Furuse M, Hirase T, Itoh M, Nagafuchi A, Yonemura S, Tsukita S. Occludin: a novel integral membrane protein localizing at tight junctions. J Cell Biol 1993;123:1777-1788.

14. Furuse M, Fujita K, Hiiragi T, Fujimoto K, Tsukita S. Claudin-1 and -2: novel integral membrane proteins localizing at tight junctions with no sequence similarity to occludin. J Cell Biol 1998;141:1539-1550.
15. Martín-Padura I, Lostaglio S, Schneemann M, et al. Junctional adhesion molecule, a novel member of the immunoglobulin superfamily that distributes at intercellular junctions and modulates monocyte transmigration. J Cell Biol 1998;142:117-127.

16. Ikenouchi J, Furuse M, Furuse K, Sasaki H, Tsukita S. Tricellulin constitutes a novel barrier at tricellular contacts of epithelial cells. J Cell Biol 2005;171:939-945.

17. González-Mariscal L, Betanzos A, Nava P, Jaramillo BE. Tight junction proteins. Prog Biophys Mol Biol 2003;81:1-44.

18. Turner JR, Rill BK, Carlson SL, et al. Physiological regulation of epithelial tight junctions is associated with myosin light-chain phosphorylation. Am J Physiol 1997;273:C1378-C1385.

19. Walsh SV, Hopkins AM, Chen J, Narumiya S, Parkos CA, Nusrat A. Rho kinase regulates tight junction function and is necessary for tight junction assembly in polarized intestinal epithelia. Gastroenterology 2001;121:566-579.

20. Furuse M, Itoh M, Hirase T, Nagafuchi A, Yonemura S, Tsukita $\mathrm{S}$. Direct association of occludin with ZO-1 and its possible involvement in the localization of occludin at tight junctions. J Cell Biol 1994;127:1617-1626.

21. Al-Sadi R, Khatib K, Guo S, Ye D, Youssef M, Ma T. Occludin regulates macromolecule flux across the intestinal epithelial tight junction barrier. Am J Physiol Gastrointest Liver Physiol 2011;300:G1054-G1064.

22. Sakakibara A, Furuse M, Saitou M, Ando-Akatsuka Y, Tsukita S. Possible involvement of phosphorylation of occludin in tight junction formation. J Cell Biol 1997;137:1393-1401.

23. Rao R. Occludin phosphorylation in regulation of epithelial tight junctions. Ann N Y Acad Sci 2009;1165:62-68.

24. Jain S, Suzuki T, Seth A, Samak G, Rao R. Protein kinase C $\zeta$ phosphorylates occludin and promotes assembly of epithelial tight junctions. Biochem J 2011;437:289-299.

25. McKenzie JA, Riento K, Ridley AJ. Casein kinase I epsilon associates with and phosphorylates the tight junction protein occludin. FEBS Lett 2006;580:2388-2394.

26. Elias BC, Suzuki T, Seth A, et al. Phosphorylation of Tyr-398 and Tyr-402 in occludin prevents its interaction with ZO-1 and destabilizes its assembly at the tight junctions. J Biol Chem 2009; 284:1559-1569.

27. Atkinson KJ, Rao RK. Role of protein tyrosine phosphorylation in acetaldehyde-induced disruption of epithelial tight junctions. Am J Physiol Gastrointest Liver Physiol 2001;280:G1280-G1288.

28. Tsukita S, Furuse M. Pores in the wall: claudins constitute tight junction strands containing aqueous pores. J Cell Biol 2000;149: 13-16. 
29. Tamura A, Hayashi H, Imasato M, et al. Loss of claudin-15, but not claudin-2, causes Na+ deficiency and glucose malabsorption in mouse small intestine. Gastroenterology 2011;140:913923.

30. Fujibe M, Chiba H, Kojima T, et al. Thr203 of claudin-1, a putative phosphorylation site for MAP kinase, is required to promote the barrier function of tight junctions. Exp Cell Res 2004; 295:36-47.

31. Cunningham SA, Arrate MP, Rodriguez JM, et al. A novel protein with homology to the junctional adhesion molecule. Characterization of leukocyte interactions. J Biol Chem 2000;275:3475034756.

32. Bazzoni G. The JAM family of junctional adhesion molecules. Curr Opin Cell Biol 2003;15:525-530.

33. Liu Y, Nusrat A, Schnell FJ, et al. Human junction adhesion molecule regulates tight junction resealing in epithelia. J Cell Sci 2000;113:2363-2374.

34. Laukoetter MG, Nava P, Lee WY, et al. JAM-A regulates permeability and inflammation in the intestine in vivo. J Exp Med 2007; 204:3067-3076.

35. Haskins J, Gu L, Wittchen ES, Hibbard J, Stevenson BR. ZO-3, a novel member of the MAGUK protein family found at the tight junction, interacts with ZO-1 and occludin. J Cell Biol 1998;141: 199-208.

36. Willott E, Balda MS, Fanning AS, Jameson B, Van Itallie C, Anderson JM. The tight junction protein ZO-1 is homologous to the Drosophila discs-large tumor suppressor protein of septate junctions. Proc Natl Acad Sci U S A 1993;90:7834-7838.

37. Fanning AS, Ma TY, Anderson JM. Isolation and functional characterization of the actin binding region in the tight junction protein ZO-1. FASEB J 2002;16:1835-1837.

38. Umeda K, Matsui T, Nakayama M, et al. Establishment and characterization of cultured epithelial cells lacking expression of ZO-1. J Biol Chem 2004;279:44785-44794.

39. Stallmach A, Giese T, Schmidt C, Ludwig B, Mueller-Molaian I, Meuer SC. Cytokine/chemokine transcript profiles reflect mucosal inflammation in Crohn's disease. Int J Colorectal Dis 2004;19:308-315.

40. Bruewer M, Utech M, Ivanov AI, Hopkins AM, Parkos CA, Nusrat A. Interferon- $\gamma$ induces internalization of epithelial tight junction proteins via a macropinocytosis-like process. FASEB J 2005;19:923-933.

41. Schulzke JD, Bojarski C, Zeissig S, Heller F, Gitter AH, Fromm M. Disrupted barrier function through epithelial cell apoptosis. Ann N Y Acad Sci 2006;1072:288-299.
42. Ma TY, Boivin MA, Ye D, Pedram A, Said HM. Mechanism of TNF- $\alpha$ modulation of Caco-2 intestinal epithelial tight junction barrier: role of myosin light-chain kinase protein expression. Am J Physiol Gastrointest Liver Physiol 2005;288:G422-G430.

43. Mankertz J, Amasheh M, Krug SM, et al. TNF $\alpha$ up-regulates claudin-2 expression in epithelial HT-29/B6 cells via phosphatidylinositol-3-kinase signaling. Cell Tissue Res 2009;336:67-77.

44. Dinarello CA. The interleukin-1 family: 10 years of discovery. FASEB J 1994;8:1314-1325.

45. Barksby HE, Lea SR, Preshaw PM, Taylor JJ. The expanding family of interleukin-1 cytokines and their role in destructive inflammatory disorders. Clin Exp Immunol 2007;149:217-225.

46. Al-Sadi R, Ye D, Dokladny K, Ma TY. Mechanism of IL-1 $\beta$-induced increase in intestinal epithelial tight junction permeability. J Immunol 2008;180:5653-5661.

47. Al-Sadi R, Ye D, Said HM, Ma TY. IL- $1 \beta$-induced increase in intestinal epithelial tight junction permeability is mediated by MEKK-1 activation of canonical NF- $\kappa$ B pathway. Am J Pathol 2010;177:2310-2322.

48. Kusugami K, Fukatsu A, Tanimoto M, et al. Elevation of interleukin-6 in inflammatory bowel disease is macrophage- and epithelial cell-dependent. Dig Dis Sci 1995;40:949-959.

49. Suzuki T, Yoshinaga N, Tanabe S. Interleukin-6 (IL-6) regulates claudin-2 expression and tight junction permeability in intestinal epithelium. J Biol Chem 2011;286:31263-31271.

50. Kucharzik T, Lugering N, Pauels HG, Domschke W, Stoll R. IL4, IL-10 and IL-13 down-regulate monocyte-chemoattracting protein-1 (MCP-1) production in activated intestinal epithelial cells. Clin Exp Immunol 1998;111:152-157.

51. Madsen KL, Malfair D, Gray D, Doyle JS, Jewell LD, Fedorak RN. Interleukin-10 gene-deficient mice develop a primary intestinal permeability defect in response to enteric microflora. Inflamm Bowel Dis 1999;5:262-270.

52. Kansagra K, Stoll B, Rognerud C, et al. Total parenteral nutrition adversely affects gut barrier function in neonatal piglets. Am J Physiol Gastrointest Liver Physiol 2003;285:G1162-G1170.

53. Sun X, Yang H, Nose K, et al. Decline in intestinal mucosal IL-10 expression and decreased intestinal barrier function in a mouse model of total parenteral nutrition. Am J Physiol Gastrointest Liver Physiol 2008;294:G139-G147.

54. Pappu R, Ramirez-Carrozzi V, Sambandam A. The interleukin-17 cytokine family: critical players in host defence and inflammatory diseases. Immunology 2011;134:8-16.

55. Kinugasa T, Sakaguchi T, Gu X, Reinecker HC. Claudins regulate the intestinal barrier in response to immune mediators. Gastroenterology 2000;118:1001-1011. 
56. Basuroy S, Seth A, Elias B, Naren AP, Rao R. MAPK interacts with occludin and mediates EGF-induced prevention of tight junction disruption by hydrogen peroxide. Biochem J 2006;393: 69-77.

57. Samak G, Aggarwal S, Rao RK. ERK is involved in EGF-mediated protection of tight junctions, but not adherens junctions, in acetaldehyde-treated Caco-2 cell monolayers. Am J Physiol Gastrointest Liver Physiol 2011;301:G50-G59.

58. Howe KL, Reardon C, Wang A, Nazli A, McKay DM. Transforming growth factor- $\beta$ regulation of epithelial tight junction proteins enhances barrier function and blocks enterohemorrhagic Escherichia coli O157:H7-induced increased permeability. Am J Pathol 2005;167:1587-1597.

59. Söderholm JD, Peterson KH, Olaison G, et al. Epithelial permeability to proteins in the noninflamed ileum of Crohn's disease? Gastroenterology 1999;117:65-72.

60. Zeissig S, Bürgel N, Günzel D, et al. Changes in expression and distribution of claudin 2, 5 and 8 lead to discontinuous tight junctions and barrier dysfunction in active Crohn's disease. Gut 2007;56:61-72.

61. Vetrano S, Rescigno M, Cera MR, et al. Unique role of junctional adhesion molecule-a in maintaining mucosal homeostasis in inflammatory bowel disease. Gastroenterology 2008;135:173184.
62. Blair SA, Kane SV, Clayburgh DR, Turner JR. Epithelial myosin light chain kinase expression and activity are upregulated in inflammatory bowel disease. Lab Invest 2006;86:191-201.

63. Murch SH, Braegger CP, Walker-Smith JA, MacDonald TT. Location of tumour necrosis factor alpha by immunohistochemistry in chronic inflammatory bowel disease. Gut 1993;34:1705-1709.

64. Louis E, Belaiche J, van Kemseke C, et al. A high serum concentration of interleukin-6 is predictive of relapse in quiescent Crohn's disease. Eur J Gastroenterol Hepatol 1997;9:939-944.

65. Suenaert P, Bulteel V, Lemmens L, et al. Anti-tumor necrosis factor treatment restores the gut barrier in Crohn's disease. Am J Gastroenterol 2002;97:2000-2004.

66. Schmitz H, Fromm M, Bentzel CJ, et al. Tumor necrosis factoralpha $(\mathrm{TNF} \alpha)$ regulates the epithelial barrier in the human intestinal cell line HT-29/B6. J Cell Sci 1999;112 ( Pt 1):137-146.

67. Wang F, Graham WV, Wang Y, Witkowski ED, Schwarz BT, Turner JR. Interferon- $\gamma$ and tumor necrosis factor- $\alpha$ synergize to induce intestinal epithelial barrier dysfunction by up-regulating myosin light chain kinase expression. Am J Pathol 2005;166: 409-419.

68. Graham WV, Wang F, Clayburgh DR, et al. Tumor necrosis factor-induced long myosin light chain kinase transcription is regulated by differentiation-dependent signaling events. Characterization of the human long myosin light chain kinase promoter. J Biol Chem 2006;281:26205-26215. 